\title{
Quantum mechanical analysis of the equilateral triangle billiard: periodic orbit theory and wave packet revivals
}

\author{
M. A. Doncheski \\ Department of Physics \\ The Pennsylvania State University \\ Mont Alto, PA 17237 USA \\ R. W. Robinett \\ Department of Physics \\ The Pennsylvania State University \\ University Park, PA 16802 USA
}

\begin{abstract}
Using the fact that the energy eigenstates of the equilateral triangle infinite well (or billiard) are available in closed form, we examine the connections between the energy eigenvalue spectrum and the classical closed paths in this geometry, using both periodic orbit theory and the short-term semi-classical behavior of wave packets. We also discuss wave packet revivals and show that there are exact revivals, for all wave packets, at times given by $T_{\text {rev }}=9 \mu a^{2} / 4 \hbar \pi$ where $a$ and $\mu$ are the length of one side and the mass of the point particle respectively. We find additional cases of exact revivals with shorter revival times for zero-momentum wave packets initially located at special symmetry points inside the billiard. Finally, we discuss simple variations on the equilateral $\left(60^{\circ}-60^{\circ}-60^{\circ}\right)$ triangle, such as the half equilateral $\left(30^{\circ}-60^{\circ}-90^{\circ}\right)$ triangle and other 'foldings', which have related energy spectra and revival structures.
\end{abstract}




\section{INTRODUCTION}

The study of the connections between the quantized energy eigenvalues of a bound state and the classical motions of the corresponding classical point particle has undergone something of a renaissance as the ability to experimentally probe the quantum-classical interface has dramatically improved. Theoretical methods such as periodic orbit theory [1], 2], [3], for example, provide very direct connections between the energy spectrum and the closed trajectories of the classical system. The time-dependence of fully quantum mechanical wave packet solutions of the Schrödinger equation also depends critically on the energy spectrum, both for the short-term semi-classical propagation as well as for longer-term, purely quantum mechanical effects such as wave packet revivals. Such revival phenomena have been observed in a wide variety of physical systems, especially in Rydberg atoms [4], and calculations exist for many other systems [5].

Two-dimensional billiard systems have provided easily visualizible examples relevant for both types of analyses. For example, the periodic orbit theory analysis of square/rectangular 6] and spherical/circular [7] geometries were among the first performed, while on the experimental side, the energy level structure and statistics of microwave cavities [8] (modeling 2D billiards of arbitrary shape) have been probed to test both periodic orbit theory and to obtain statistical evidence of chaotic behavior. Measurements of conductance fluctuations in ballistic microstructures [9] have been tentatively used to identify frequency features in the power spectrum with specific closed orbits in a circular (and stadium) billiard. More recently, the realization of atom-optics billiards [10], with ultra-cold atoms in arbitrary shaped 2D boundaries confined by optical dipole potentials, has allowed the study of various chaotic and integrable shapes such as the stadium, ellipse and circle, again for short-term, semi-classical propagation.

The one- [11] and two-dimensional [12], 13] square wells, with their integral energy spectra, have provided simple examples of exact wave packet revivals, wherein initially localized states which have a short-term, quasi-classical time evolution, can then spread significantly over several orbits, only to reform later in the form of a quantum revival in which the spreading reverses itself, the wave packet relocalizes, and the semi-classical periodicity is once again evident. Circular billiards have also been recently studied [14] where approximate revivals have been found to be present under some circumstances. 
One billiard system which has not been analyzed in as much detail is the case of the equilateral (or $60^{\circ}-60^{\circ}-60^{\circ}$ ) triangle quantum infinite well or billiard. If one thinks of the square billiard as an $N=4$ regular polygon, with the circle being the $N \rightarrow \infty$ limit, the $N=3$ case of an equilateral triangular billiard is an obvious extension. Since the exact bound state wavefunctions for the corresponding infinite well problem have been available for some time [15], [16], 17], our purpose here will be to apply these known results and analyze the quantum mechanics of this system in the context of periodic orbit theory as well as wave packet revivals. Given how few quantum systems, especially billiard geometries, have closed-form solutions, it is very useful to have another such example analyzed in as much detail as possible. In addition, the energy eigenvalues for this case, described by simple quadratic powers of quantum numbers, just as for the 2D square billiard, will be seen to yield exact quantum revivals, providing another interesting 'testbed' for possible future experimental tests.

In Section II, we review the solutions of the equilateral triangular well, wavefunctions and energy eigenvalues, and make some comments about simple variations resulting from various 'foldings' of the standard equilateral triangle billiard. We then perform a periodic orbit theory analysis of this system in Sec. III and discuss the exact wave packet revivals present in this system in Sec. IV, as well as visualizing the short-term semiclassical propagation wave packets and their relationship to classical closed orbits.

\section{ENERGY EIGENFUNCTIONS AND EIGENVALUES}

\section{A. Results for the equilateral triangle billiard}

The energy eigenvalues and position-space wave functions for a particle of mass $\mu$ in an equilateral triangular infinite well (or billiard) of side $a$ have been derived, in a variety of different contexts, by at least three different groups [15], 16], 17]. For definiteness, we will assume a triangular billiard with vertices located at $(0,0),(a / 2, \sqrt{3} a / 2)$, and $(-a / 2, \sqrt{3} a / 2)$, as shown in Fig. 1. The resulting energy spectrum is given by

$$
E(m, n)=\frac{\hbar^{2}}{2 \mu a^{2}}\left(\frac{4 \pi}{3}\right)^{2}\left(m^{2}+n^{2}-m n\right)
$$

for integral values of $m, n$, with the restriction that $m \geq 2 n$. (In what follows, we will use the notations of Ref. [16] for the energies and wavefunctions: we use $\mu$ for the particle mass 
to avoid confusion with various quantum numbers.) For the case of $m>2 n$, there are two degenerate states with different symmetry properties [16] which can be written in the forms

$$
\begin{aligned}
\psi_{(m, n)}^{(-)}(x, y)=\sqrt{\frac{16}{a^{2} 3 \sqrt{3}}} & {\left[\sin \left(\frac{2 \pi(2 m-n) x}{3 a}\right) \sin \left(\frac{2 \pi n y}{\sqrt{3} a}\right)\right.} \\
& -\sin \left(\frac{2 \pi(2 n-m) x}{3 a}\right) \sin \left(\frac{2 \pi m y}{\sqrt{3} a}\right) \\
& \left.-\sin \left(\frac{2 \pi(m+n) x}{3 a}\right) \sin \left(\frac{2 \pi(m-n) y}{\sqrt{3} a}\right)\right]
\end{aligned}
$$

and

$$
\begin{aligned}
\psi_{(m, n)}^{(+)}(x, y)=\sqrt{\frac{16}{a^{2} 3 \sqrt{3}}} & {\left[\cos \left(\frac{2 \pi(2 m-n) x}{3 a}\right) \sin \left(\frac{2 \pi n y}{\sqrt{3} a}\right)\right.} \\
& -\cos \left(\frac{2 \pi(2 n-m) x}{3 a}\right) \sin \left(\frac{2 \pi m y}{\sqrt{3} a}\right) \\
& \left.+\cos \left(\frac{2 \pi(m+n) x}{3 a}\right) \sin \left(\frac{2 \pi(m-n) y}{\sqrt{3} a}\right)\right] .
\end{aligned}
$$

Extending earlier results, we have here included the correct normalizations, since we will eventually expand Gaussian wave packets in such eigenstates.

For the special case of $m=2 n$ there is a single non-degenerate state for each $n$, given by

$$
\psi_{(2 n, n)}^{(o)}(x, y)=\sqrt{\frac{8}{a^{2} 3 \sqrt{3}}}\left[2 \cos \left(\frac{2 \pi n x}{a}\right) \sin \left(\frac{2 \pi n y}{\sqrt{3} a}\right)-\sin \left(\frac{4 \pi n y}{\sqrt{3} a}\right)\right] .
$$

Clearly these states satisfy

$$
\psi_{(m, n)}^{( \pm)}(-x, y)= \pm \psi_{(m, n)}^{( \pm)}(x, y) \quad, \quad \psi_{(m, n)}^{(o)}(-x, y)=+\psi_{(m, n)}^{(o)}(x, y)
$$

and the $\psi_{(m=2 n, n)}^{( \pm)}(x, y)$ states also satisfy

$$
\begin{aligned}
& \psi_{(m=2 n, n)}^{(+)}(x, y)=\sqrt{2} \psi_{(2 n, n)}^{(o)}(x, y) \\
& \psi_{(m=2 n, n)}^{(-)}(x, y)=0 .
\end{aligned}
$$

The authors of Ref. [16] note that the $\psi_{(m, n)}^{( \pm)}(x, y)$ states also satisfy such relations as

$$
\psi_{(m, m-n)}^{( \pm)}(x, y)= \pm \psi_{(m, n)}^{( \pm)}(x, y) \quad \text { and } \quad \psi_{(n, m)}^{( \pm)}(x, y)=-\psi_{(m, n)}^{( \pm)}(x, y)
$$

and several other similar ones. The extremely symmetric character of the $(2 n, n)$ states can also be seen by the fact that it can be rewritten in the form

$$
\psi_{(2 n, n)}^{(o)}(x, y)=\frac{8 \sqrt{2}}{3^{3 / 4} a} \sin \left(\frac{2 \pi n y}{\sqrt{3} a}\right) \sin \left(\frac{\pi n(y-\sqrt{3} x)}{\sqrt{3} a}\right) \sin \left(\frac{\pi n(y+\sqrt{3} x)}{\sqrt{3} a}\right) .
$$


These wavefunctions satisfy the Schrödinger equation with the energy eigenvalues in Eqn. (1), as well as vanishing on the boundaries defined by the lines $y= \pm \sqrt{3} x$ and $y=\sqrt{3} a / 2$. The eigenfunctions corresponding to different eigenvalues can be shown to be orthogonal, while the two with the same eigenvalues $\left(\phi_{(m, n)}^{( \pm)}(x, y)\right)$ are obviously so due to their $x \rightarrow-x$ parity properties. The authors of both Ref. [15] and [16] argue that the solutions above also form a complete set of states by explicitly calculating the Weyl area rule for the energy level density

$$
\rho_{0}(E)=\frac{A}{4 \pi}\left(\frac{2 \mu}{\hbar^{2}}\right)-\frac{L}{8 \pi} \sqrt{\frac{2 \mu}{\hbar^{2} E}}
$$

and showing that this relation is saturated by the energy spectrum in Eqn. (11). While we will focus on the case of the equilateral triangle billiard, it is useful to note that several simple variations on this system (see below) can also be derived from these results.

We note that these solutions, while derived in the context of the explicit triangular area shown in Fig. 1 (shown in bold), are also solutions, when extended by reflections,

in the other triangular regions (flipped in sign) and hence over the complete hexagonal area including reflections in the horizontal axis. (This connection, in fact, was used in the explicit construction of the solutions in one approach [16].) Thus, these solutions (with their integrally quantized energies, will also form a subset of the solutions for the hexagonal $(N=6$ regular polygonal) billiard.

\section{B. Comparison to the square and circular billiards}

The degeneracy pattern of energy eigenvalues for the equilateral triangle billiard is very similar to that for the square $(N=4$ polygon $)$ and circular $(N=\infty$ polygon) systems. For the square billiard (of side $a$ ), the energy eigenvalues are given by

$$
E(m, n)=\frac{\hbar^{2} \pi^{2}}{2 \mu a^{2}}\left(m^{2}+n^{2}\right)
$$

with eigenfunctions given by

$$
\psi_{(m, n)}(x, y)=u_{m}(x) u_{n}(y)
$$

with

$$
u_{n}(x)=\sqrt{\frac{2}{a}} \sin \left(\frac{n \pi x}{a}\right)
$$


and any integral $m, n \geq 1$ allowed. For $m=n$, there is a single state, while for $m \neq n$ there is a two-fold degeneracy. The analogs of the degenerate $( \pm)$ states of the equilateral triangle billiard for the square infinite well are given by

$$
\begin{array}{ll}
\psi_{(m, n)}^{(-)}(x, y)=\frac{1}{\sqrt{2}}\left[u_{n}(x) u_{m}(y)-u_{m}(x) u_{n}(y)\right] & (m \neq n) \\
\psi_{(m, n)}^{(+)}(x, y)=\frac{1}{\sqrt{2}}\left[u_{n}(x) u_{m}(y)+u_{m}(x) u_{n}(y)\right] \quad(m \neq n) \\
\psi_{(n, n)}^{(o)}(x, y)=u_{n}(x) u_{n}(y) .
\end{array}
$$

This form is useful since it allows one to discuss the energy eigenvalues and eigenfunctions of the $45^{\circ}-45^{\circ}-90^{\circ}$ triangle [19] billiard formed by 'folding' the square along a diagonal [18], since the $\psi_{(m, n)}^{(-)}(x, y)$ satisfy the appropriate boundary condition along the new hypotenuse. Additional foldings along diagonals are also possible [18] and the energy spectrum can be used to analyze these cases as well.

A similar pattern of degeneracies, also useful for analyzing 'half-well' problems, is found in the circular well [18], [20]. In this case, the wavefunctions in polar coordinates can be written in the form

$$
\psi(r, \theta)=\left[N_{r} J_{|m|}(k r)\right]\left[N_{\theta} e^{i m \theta}\right] \quad \text { where } m=0, \pm 1, \pm 2, \ldots
$$

where the radial wavefunctions are normalized via

$$
\int_{0}^{R}\left[N_{r} J_{|m|}(k r)\right]^{2} r d r=1 .
$$

The states corresponding to non-negative values of $+m$ and $-m$ are degenerate. A standard set of angular wavefunctions can be written as

$$
\int_{0}^{2 \pi}\left|N_{\theta} e^{i m \theta}\right|^{2} d \theta=1 \quad \text { or } \quad N_{\theta}=1 / \sqrt{2 \pi},
$$

but for the purposes of visualizing the solutions of bound state problems, real wavefunctions are also possible, given by

$$
\Theta_{|m|}(\theta)=\left\{\begin{array}{ll}
\cos (m \theta) / \sqrt{\pi} & m \geq 1 \\
\sin (m \theta) / \sqrt{\pi} & m \geq 1 . \\
1 / \sqrt{2 \pi} & m=0
\end{array} .\right.
$$

Thus, there is a doubly degenerate set of states labeled by $\left(m>1, n_{r}\right)$ for $m>1$, and a single set of states (all of the $s$-state wavefunctions) with $\left(m=0, n_{r}\right)$. One of the $\left(m>1, n_{r}\right)$ 
sets can then be used to describe the energies of the 'half-circular' billiard, as either the $\cos (m \theta)$ or $\sin (m \theta)$ solutions (or some linear combination thereof) can be used to satisfy the boundary condition along an infinite wall on any diameter, giving a 'folded half-well'.

\section{Variants on the equilateral triangle}

Using the explicit wavefunctions for equilateral triangle, it is easy to construct solutions of the 'half-triangle' (namely the $30^{\circ}-60^{\circ}-90^{\circ}$ triangle) obtained by folding the equilateral triangle along one bisector: the odd-parity solutions in Eqn. (2), for example, explicitly satisfy the new boundary condition along the bisector given by $x=0$. The resulting energies are still given by Eqn. (11), but now with only a single copy of the $m>2 n$ states allowed, which agrees with earlier analyses [15], 19]. The wavefunctions in the new 'half-well' are

identical to the $\psi_{(m, n}^{(-)}(x, y)$ except with an additional normalization factor of $\sqrt{2}$ to account for the geometrical 'footprint' of the smaller billiard.

The three lowest-lying such states, corresponding to $(m, n)$ quantum numbers and energy values of $(3,1),(4,1)$, and $(5,2)$ and $7 E_{0}, 13 E_{0}$, and $19 E_{0}$ respectively (where $E_{0} \equiv$ $\left.\left(\hbar^{2} / 2 \mu a^{2}\right)(4 \pi / 3)^{2}\right)$ are shown on the top line of Fig. 2.

It is easy to shown that if one lets $\left(m^{\prime}, n^{\prime}\right)=(2 m, 2 n)$ then all of the energy eigenvalues are multiplied by the same factor of 4 and that there are nodal lines which split up each 'half-triangle' into four equal parts corresponding to a simple folding. The corresponding states for this subset of quantum numbers are also shown in Fig. 2 (in the bottom row). This possibility is part of a general pattern where the mapping $\left(m^{\prime}, n^{\prime}\right)=(f m, f n)$ yields $f^{2}$ 'copies' of the $(m, n)$ wavefunctions (for both the even and odd states) contained inside the original triangular billiard; one can see this more explicitly by noting that

$$
\psi_{(f m, f n)}^{( \pm)}(x, y ; a)=\psi_{(m, n)}^{( \pm)}(x, y ; a / f) .
$$

Slightly less trivially, the quantum number mapping given by $\left(m^{\prime}, n^{\prime}\right)=(2 m-n, m-2 n)$ gives states which are a factor of 3 times in energy, with nodal lines along the perpendicular bisectors, as shown in (the middle row of) Fig. 2. A second application of this map gives $\left(m^{\prime \prime}, n^{\prime \prime}\right)=(3 m, 3 n)$ and a resulting common increase by a factor of 9 , as mentioned above. Both sets of results can be then be easily understood in terms of repeated foldings of the 'half-triangle' onto itself, with increasingly small areas and larger energy eigenvalues scaling 
in the appropriate manner, just as in Ref. [18] for diagonal foldings of the square billiard. For this case, we might note that the odd wavefunctions explicitly satisfy the relation

$$
\psi_{(2 m-n, m-2 n)}^{(-)}(x, y ; a)=-\psi_{(m, n)}^{(-)}(y, x ; a / 3) .
$$

Other simple linear transformations of quantum numbers of the form $\left(m^{\prime}, n^{\prime}\right)=(\alpha m+$ $\beta n, \gamma m+\delta n)$ also return allowed quantum numbers in the triangular well, corresponding to higher energy states, but without the same obvious geometrical significance. A few examples of such mappings are discussed briefly in the Appendix.

\section{PERIODIC ORBIT THEORY ANALYSIS}

\section{A. General background}

As mentioned above, the notion of density of energy levels has already been applied to the solutions of triangular billiard to the extent that the averaged energy level density, $\rho_{0}(E)$, in Eqn. (92), has been evaluated to confirm completeness, as in Refs. [15] and [16]. It is perhaps then an obvious next step to expand on this type of approach by using a periodic orbit

theory analysis [1], 2], 3]. In this context, the energy level density is naturally split into the smooth, slowly varying part $\left(\rho_{0}(E)\right)$ and a second, oscillatory term which is dominated (in a saddle point approximation of the path integral) by classical orbits whose actions, $S_{\gamma}(E)$, correspond to periodic orbits or closed paths. Specifically, using the quantized energy eigenvalues, labeled collectively as $E_{n}$, one writes

$$
\sum_{n=1}^{\infty} \delta\left(E-E_{n}\right) \equiv \rho(E)=\rho_{0}(E)+\sum_{p=1}^{\infty} \sum_{\gamma} \rho_{\gamma, p} \cos \left[p\left(\frac{S_{\gamma}(E)}{\hbar}-\phi_{\gamma}\right)\right]
$$

where each periodic orbit is characterized by a label $\gamma$ and $p=1,2, \ldots$ runs over all possible repetitions of such trajectories. For billiard systems, this expression can be Fourier transformed and simplified in terms of

$$
\rho(L) \equiv \sum_{n=1}^{\infty} \int_{-\infty}^{+\infty} \delta\left(k-k_{n}\right) e^{i k L} d k=\sum_{n=1}^{\infty} e^{i k_{n} L}
$$

to give

$$
\rho(L)=\sum_{p=1}^{\infty} \sum_{\gamma} \rho_{\gamma, p} \delta\left(L-p L_{\gamma}\right)
$$


Thus, an evaluation of $\rho(L)$, using the quantized $k_{n}$ values given by the $E_{n}=\hbar^{2} k_{n}^{2} / 2 \mu$, will exhibit a series of $\delta$-function like 'spikes' at multiples of the lengths of the 'primitive' closed paths, i.e., at $L=p L_{\gamma}$. For numerical studies, an evaluation of

$$
\rho_{N}(L)=\sum_{n=1}^{N} e^{i k_{n} L}
$$

for large, but finite $N$ will give a series of sharp peaks corresponding to the closed orbits, with the smooth $\rho_{0}(E)$ piece corresponding to a large, but uninteresting feature at $L=0$.

\section{B. Periodic orbit theory analysis of the equilateral triangle billiard}

For a numerical evaluation of $\rho_{N}(L)$, we require the quantized wavenumbers obtained from the energy spectrum in Eqn. (11), namely

$$
k_{(m, n)} a=\left(\frac{4 \pi}{3}\right) \sqrt{m^{2}+n^{2}-m n}
$$

so that

$$
\left\{k_{(m, n)} ; m / 2 \geq n \geq 1\right\}=\{7.255,11.082,11.082,14.510,15.102,15.102, \ldots\}
$$

where including the correct degeneracy, as we will see, is important. An analysis of $\rho_{N}(L)$, evaluated using Eqn. (23), then requires knowledge of the possible closed paths or periodic orbits in the triangular billiard.

A particularly easy way to visualize the possible classes of closed orbits in this geometry is by making use of the fact that the $2 \mathrm{D}$ plane can be tiled with a triangular lattice, as illustrated in Fig. 3. Consider a specific equilateral triangle billiard, in this case the one in the lower left hand corner. By repeated foldings, the entire 2D plane can be covered, and 'equivalent' triangles are shown with appropriate points identified. Any point in the original triangle can then be connected to the corresponding point in another 'identified' partner (as illustrated by the arrows) and the resulting path inside a specific triangular billiard can be

obtained (visualized) by repeatedly folding the two triangles until they overlap. (We note that this simple construction has been used for the square billiard [21] to obtain the path lengths for the closed orbits.) In Fig. 4 we illustrate several cases corresponding to different starting points within the triangle, yielding identical overall path lengths, so long as the initial angles are the same. We note that, just as for the square and circular billiards, there 
are an infinite number of possible closed orbits of the same path length, here corresponding to different initial locations of the orbit. For example, the trajectories shown in Figs. 4(a) and (b) both correspond to path lengths of $L=3 a$ (and multiples thereof), while those in Figs. 4(d), (e), and (f) all yield path lengths of $\sqrt{3} a$. Fig. 4(c) shows a unique (isolated) orbit which, because it closes on itself in a very symmetric way, corresponds to a single possible closed orbit with path lengths given by multiples of $3 a / 2$.

The general expression for the allowed closed orbit lengths can then be obtained using this construction. For example, without loss of generality, we can find the path lengths between a single corner in the initial triangle in Fig. 3 (say the corner at $(0,0)$, labeled by the circle) and any other corresponding corner. For those points corresponding to triangles which have been folded into 'even' rows, we can, for example, use the points

$$
x_{i_{1}}=i_{1}(3 a) \quad \text { and } \quad y_{j_{1}}=j_{1}(\sqrt{3} a)
$$

which gives

$$
\begin{aligned}
d\left(i_{1}, j_{1}\right) & \equiv \sqrt{\left(x_{i_{1}}-0\right)^{2}+\left(y_{j_{1}}-0\right)^{2}} \\
& =a \sqrt{9 i_{1}^{2}+3 j_{1}^{2}}=\frac{a}{2} \sqrt{9\left(2 i_{1}\right)^{2}+3\left(2 j_{1}\right)^{2}}
\end{aligned}
$$

This corresponds to initial angles (with respect to the horizontal) of

$$
\tan (\theta)=\frac{y_{j_{1}}}{x_{i_{1}}}=\frac{j_{1}}{i_{1} \sqrt{3}}
$$

For points in triangles which have been folded into 'odd' rows, we similarly have

$$
x_{i_{2}}=\left(2 i_{2}+1\right) \frac{3 a}{2} \quad \text { and } \quad y_{j_{2}}=\left(2 j_{2}+1\right) \frac{\sqrt{3} a}{2}
$$

giving closed path distances

$$
\begin{aligned}
d\left(i_{2}, j_{2}\right) & \equiv \sqrt{\left(x_{i_{2}}-0\right)^{2}+\left(y_{j_{2}}-0\right)^{2}} \\
& =\frac{a}{2} \sqrt{9\left(2 i_{2}+1\right)^{2}+3\left(2 j_{2}+1\right)^{2}}
\end{aligned}
$$

and initial angles

$$
\tan (\theta)=\frac{y_{j_{1}}}{x_{i_{1}}}=\frac{\left(2 j_{2}+1\right)}{\left(2 i_{2}+1\right) \sqrt{3}} .
$$

We can combine these two cases into a single expression, namely

$$
d(\bar{i}, \bar{j})=\frac{a}{2} \sqrt{9(\bar{i})^{2}+3(\bar{j})^{2}}
$$


where $(\bar{i}, \bar{j})$ are either both even or both odd. The corresponding angles are given by

$$
\tan (\theta)=\frac{\bar{j}}{\bar{i} \sqrt{3}} .
$$

The path length features for these classes of closed orbits (and their recurrences) which have $L=d(\bar{i}, \bar{j})<20 a$, along with the corresponding $(\bar{i}, \bar{j})$ labels and angles, are shown in Table I. This construction finds all of the allowed closed orbits, except for the special case described by Fig. 4(c).

We can rewrite Eqn. (32) in a more symmetric form by defining

$$
(p+q) \equiv \bar{i} \quad, \quad(p-q) \equiv \bar{j} \quad \text { or } \quad p \equiv \frac{\bar{i}+\bar{j}}{2} \quad, \quad q \equiv \frac{\bar{i}-\bar{j}}{2}
$$

Since $\bar{i}, \bar{j}$ are either both even or both odd, the $p, q$ defined in this way are integer valued and we find that the path lengths can be written as

$$
d(p, q)=a \sqrt{3} \sqrt{p^{2}+p q+q^{2}}
$$

and this form will be useful when we discuss how the closed or periodic orbits arise in the context of wave packet propagation in the next Section.

We then evaluate $\rho_{N}(L)$ (using the $N=1000$ lowest lying eigenvalues) and plot $\left|\rho_{N}(L)\right|^{2}$ versus $0 \leq L / a \leq 20$ in Fig. 5. The path length features collected in Table I are shown as vertical dotted lines and the vertical scale has been chosen to emphasize these features. We note also that there are two much smaller features, indicated by arrows, corresponding to the isolated, single closed orbit trajectories at the first two odd multiples of $L / a=3 / 2$, namely $L / a=1.5$ (denoted by $\left.(\bar{i}, \bar{j})=(2,0)^{\prime}\right)$ and $L / a=4.5\left(\right.$ for $\left.3(2,0)^{\prime}\right)$. Even recurrences are, of course, hidden under the standard $((\bar{i}, \bar{j})=(2,0)) L / a=3.0$ orbits, while higher odd recurrences are close to other features corresponding to different orbits: for example the $5(2,0)^{\prime}$ feature at $L / a=7.5$ is near a more prominent $(5,1)$ feature at $L / a=7.55$, while the $L / a=10.5,7(2,0)^{\prime}$ feature is shadowed by the $L / a=10.53(7,1)$ orbit.

\section{Periodic orbit theory analysis of the 'half-triangle'}

We can easily extend the analysis of the equilateral triangle billiard to the 'half-triangle' or $30^{\circ}-60^{\circ}-90^{\circ}$ triangle by using the reduced set of $(m>2 n)$ energy eigenvalues corresponding

to the $\psi_{(m, n)}^{(-)}(x, y)$ states which vanish along the $y$-axis as discussed in Section II C and the 
evaluation of $\rho_{N}(L)$ proceeds just as above. The only substantiative changes in the allowed classical closed orbits can be visualized as has been done for various foldings of the square and circular wells in Ref. [18]. For all allowed orbits (save one special one), the introduction of an infinite wall along a bisector simply serves to reflect all trajectories back onto themselves inside the half-triangle, giving identical overall path lengths for closed orbits, as exemplified in Figs. 6(a) and (b) for two of the $(\bar{i}, \bar{j})=(1,1)$ orbits. In this case only, there is a second isolated path (shown in Fig. 6(c)) with half the expected path length, namely $L / a=\sqrt{3} a / 2$ (and multiples thereof) which we will call the $(1,1)^{\prime}$ orbit.

We thus expect to see in the $\left|\rho_{N}(L)\right|^{2}$ spectrum the same path length features as for the full-triangle, but with much smaller new features at odd multiples of $L / a=\sqrt{3} / 2$, or $L / a \approx 0.866,2.598,4.330,6.062,7.794,9.526, \ldots$ and so forth. We plot, in Fig. $7, \rho_{N}(L)$ for both the full-well (dashed) and half-well (solid) cases, adjusting the overall scales to emphasize the similar shape, and we note the agreement at all of the standard, non-isolated path length features in Table I, as well as for the isolated $L / a=3 / 2(2,0)^{\prime}$ orbit. For the half-well data, however, we also see clear evidence for all of the new isolated $(1,1)^{\prime}$ orbit features (shown by arrows.)

\section{TIME-DEPENDENCE OF GAUSSIAN WAVE PACKETS}

\section{A. General time-dependence of wave packet solutions}

The connections between the energy eigenvalue spectrum of a quantum system and the classical dynamics of particles can also be explored through the time-dependence of wave packet solutions of the Schrödinger equation. For a localized wave packet in a system characterized by a single quantum number, $n$, an arbitrary wave packet solution can be written in the form

$$
\psi(x, t)=\sum_{n=1}^{\infty} a_{n} \psi_{n}(x) e^{-i E(n) t / \hbar} .
$$

One then typically expands the energy eigenvalues (assuming integral values) about the central value, $n_{0}$, used in the construction of the wave packet to write

$$
E(n) \approx E\left(n_{0}\right)+E^{\prime}\left(n_{0}\right)\left(n-n_{0}\right)+\frac{1}{2} E^{\prime \prime}\left(n_{0}\right)\left(n-n_{0}\right)^{2}+\frac{1}{6} E^{\prime \prime \prime}\left(n_{0}\right)\left(n-n_{0}\right)^{3}+\cdots
$$


in terms of which the classical period, revival, and superrevival times [11] are given respectively by

$$
T_{c l}=\frac{2 \pi \hbar}{\left|E^{\prime}\left(n_{0}\right)\right|} \quad T_{\text {rev }}=\frac{2 \pi \hbar}{\left|E^{\prime \prime}\left(n_{0}\right)\right| / 2} \quad T_{\text {super }}=\frac{2 \pi \hbar}{\left|E^{\prime \prime \prime}\left(n_{0}\right)\right| / 6} .
$$

For systems with two quantum numbers [12], [13], and energies labeled by $E\left(n_{1}, n_{2}\right)$, the patterns of both classical periodicities and revival times can be much richer as we will see below. We first examine how the closed orbits for the equilateral triangle in Eqn. (32) arise in this formalism.

\section{B. Short-term, semi-classical periodicity in the equilateral triangle billiard}

For a system, such as the 2D billiard, for which the quantized energies depend on two quantum numbers, $(m, n)$, there are two corresponding classical periods [12], 13] given by

$$
T_{c l}^{(m)}=\frac{2 \pi \hbar}{\partial E(m, n) / \partial m} \quad, \quad T_{c l}^{(n)}=\frac{2 \pi \hbar}{\partial E(m, n) / \partial n} .
$$

Classical periodic orbits will arise when the ratio of these two times is a rational number, or equivalently when

$$
p T_{c l}^{(m)}=T_{c l}^{(p o)}=q T_{c l}^{(n)}
$$

which defines the classical period, $T_{c l}^{(p o)}$, for such closed orbits. For the equilateral triangle billiard, with energies given by

$$
E(m, n)=\frac{\hbar^{2}}{2 \mu a^{2}}\left(\frac{4 \pi}{3}\right)^{2}\left(m^{2}+n^{2}-m n\right)
$$

we find that

$$
T_{c l}^{(m)}=\left[\frac{9 \mu a^{2}}{4 \hbar \pi}\right] \frac{1}{(2 m-n)} \quad, \quad T_{c l}^{(n)}=\left[\frac{9 \mu a^{2}}{4 \hbar \pi}\right] \frac{1}{(2 n-m)} .
$$

The condition leading to closed orbits in Eqn. (40) can then be written as

$$
\frac{(2 m-n)}{(2 n-m)}=\frac{T_{c l}^{(n)}}{T_{c l}^{(m)}}=\frac{p}{q} \quad \text { or } \quad n=m\left(\frac{2 p+q}{2 q+p}\right) .
$$

If we substitute this condition into the energy spectrum in Eqn. (41), as well as equating the quantum energies with the classical kinetic energy, $\mu v_{0}^{2} / 2$, in the billiard (where $v_{0}$ is the classical speed) we are led to the association

$$
\frac{1}{2} \mu v_{0}^{2} \longleftrightarrow E(m, n)=\left(\frac{16 \pi^{2}}{9}\right)\left(\frac{\hbar^{2}}{2 \mu a^{2}}\right)\left[m^{2}+m^{2}\left(\frac{2 p+q}{2 q+p}\right)^{2}-m^{2}\left(\frac{2 p+q}{2 q+p}\right)\right]
$$


or

$$
\left(\frac{2 \mu v_{0} a}{4 \pi \hbar}\right)^{2}=m^{2}\left[\frac{3\left(p^{2}+p q+q^{2}\right)}{(2 q+p)^{2}}\right] .
$$

This implies that

$$
m=\left(\frac{2 \mu v_{0} a}{4 \pi \hbar}\right) \frac{(2 q+p)}{\sqrt{3} \sqrt{p^{2}+p q+q^{2}}} \quad, \quad n=\left(\frac{2 \mu v_{0} a}{4 \pi \hbar}\right) \frac{(2 p+q)}{\sqrt{3} \sqrt{p^{2}+p q+q^{2}}} .
$$

The period for classical, closed/periodic orbits is then given by Eqn. (40) as

$$
T_{c l}^{(p o)}=p T_{c l}^{(m)}=\frac{a \sqrt{3} \sqrt{p^{2}+p q+q^{2}}}{v_{0}}=\frac{L(p, q)}{v_{0}}
$$

where

$$
L(p, q)=d(p, q)=a \sqrt{3} \sqrt{p^{2}+p q+q^{2}}
$$

and we have used the alternative form of the path lengths for periodic orbits in Eqn. (35). (A similar analysis for the circular billiard can be found in Ref. [14], making use of the WKB approximation for the non-integral energies found there, while the corresponding derivation for the square billiard is entirely straightforward.)

To visualize this result, we make use of a specific form for Gaussian wave packets, namely

$$
\psi(x, y ; t=0)=\psi_{0}\left(x ; x_{0}, p_{0 x}, b\right) \psi_{0}\left(y ; y_{0}, p_{0 y}, b\right)
$$

where

$$
\psi_{0}\left(x ; x_{0}, p_{0 x}, b\right)=\frac{1}{\sqrt{b \sqrt{\pi}}} e^{i p_{0 x}\left(x-x_{0}\right) / \hbar} e^{-\left(x-x_{0}\right)^{2} / 2 b^{2}}
$$

with a similar expression for $\psi_{0}\left(y ; y_{0}, p_{0 y}, b\right)$.

The initial expectation values for the $x$ variables are given by

$$
\langle x\rangle_{0}=x_{0} \quad, \quad\left\langle x^{2}\right\rangle_{0}=x_{0}^{2}+\frac{b^{2}}{2} \quad, \quad \Delta x_{0}=\frac{b}{\sqrt{2}}
$$

and

$$
\left\langle p_{x}\right\rangle_{0}=p_{0 x} \quad, \quad\left\langle p_{x}^{2}\right\rangle_{0}=p_{0 x}^{2}+\frac{\hbar^{2}}{2 b^{2}} \quad, \quad \Delta p_{0}=\frac{\hbar}{\sqrt{2} b}
$$

with similar results for $y$. The expectation value of total energy is

$$
\langle\hat{E}\rangle=\frac{1}{2 m}\left\langle\hat{p}_{x}^{2}+\hat{p}_{y}^{2}\right\rangle=\frac{1}{2 m}\left[\left(p_{0 x}\right)^{2}+\left(p_{0 y}\right)^{2}+\frac{\hbar^{2}}{b^{2}}\right] .
$$

Just as with the allowed wave functions, wave packets in the billiard must satisfy the appropriate boundary conditions at the edges of the well. However, the simple Gaussian 
form above can be used to describe an initial state so long as the initial location, $\left(x_{0}, y_{0}\right)$, is well away from the edges of the potential well, so that a negligibly (exponentially) small error is made. In that case, such a Gaussian form can be easily and reproducibly expanded in terms of eigenstates, namely

$$
\begin{gathered}
\psi(x, y ; t=0)=\sum_{m>2 n}^{\infty} \sum_{n=1}^{\infty}\left(a_{(m, n)}^{(+)} \psi_{(m, n)}^{(+)}(x, y)+a_{(m, n)}^{(-)} \psi_{(m, n)}^{(-)}(x, y)\right) \\
+\sum_{n=1}^{\infty} a_{(2 n, n)}^{(o)} \psi_{(2 n, n)}^{(o)}(x, y) .
\end{gathered}
$$

For a sharply peaked Gaussian, we can (very accurately) approximate the required expansion coefficients by extending the required integration of $(x, y)$ over the triangular region to one covering all space, making use of the exact form of the wavefunctions. Given the simple results in Eqns. (2), (3) and (41), we can perform the necessary Gaussian integrals in closed form using

$$
\begin{aligned}
\int_{-\infty}^{+\infty} e^{i p_{0}\left(x-x_{0}\right) / \hbar} e^{-\left(x-x_{0}\right)^{2} / 2 b^{2}} \cos \left(\frac{C x}{a}\right) d x=\frac{b \sqrt{2 \pi}}{2} & {\left[e^{i C x_{0} / a} e^{-b^{2}\left(C / a+p_{0} / \hbar\right)^{2} / 2}\right.} \\
& \left.+e^{-i C x_{0} / a} e^{-b^{2}\left(-C / a+p_{0} / \hbar\right)^{2} / 2}\right]
\end{aligned}
$$

and

$$
\begin{aligned}
& \int_{-\infty}^{+\infty} e^{i p_{0}\left(x-x_{0}\right) / \hbar} e^{-\left(x-x_{0}\right)^{2} / 2 b^{2}} \sin \left(\frac{C x}{a}\right) d x=\frac{b \sqrt{2 \pi}}{2 i}\left[e^{i C x_{0} / a} e^{-b^{2}\left(C / a+p_{0} / \hbar\right)^{2} / 2}\right. \\
&\left.-e^{-i C x_{0} / a} e^{-b^{2}\left(-C / a+p_{0} / \hbar\right)^{2} / 2}\right] .
\end{aligned}
$$

We can then construct wave packets with arbitrary initial locations $\left(x_{0}, y_{0}\right)$ inside the well (again, so long as they are well away from any edge, typically more than a few times $\left.\Delta x_{0}=\Delta y_{0}=b / \sqrt{2}\right)$ with arbitrary initial momenta, $\left(p_{0 x}, p_{0 y}\right)$, which we parameterize via $\left(p_{0} \cos (\theta), p_{0} \sin (\theta)\right)$ to make connections with the specific angles characterizing closed orbits in Eqn. (33) and Table I.

All quantum wave packets have a characteristic spreading time, so in order to exhibit behavior typical of a semi-classical regime, we want to have classical periodicities shorter than a typical time over which a Gaussian wave packet will spread significantly. For the free-particle form in Eqn. (50), one can write

$$
\Delta x_{t}=\Delta x_{0} \sqrt{1+\left(t / t_{0}\right)^{2}} \quad \text { where } \quad t_{0} \equiv \frac{m b^{2}}{\hbar}=\frac{(2 m)}{\hbar^{2}}\left(\Delta x_{0}\right)^{2} .
$$


The classical periods for closed orbits will be given by Eqn. (47) and the two shortest such periods will be

$$
T_{c l}^{(p o)}(\min )=\frac{(\sqrt{3}, 3) a}{v_{0}}=\frac{(2 \mu) a(\sqrt{3}, 3)}{2 p_{0}}
$$

and we want to have $T_{c l}^{(p o)}(\min )<t_{0}$. For purposes of illustration then, we have taken the following numerical parameters:

$$
\hbar, 2 \mu, a=1 \quad, \quad b=1 / 10 \sqrt{2} \quad \text { so that } \quad \Delta x_{0}=\Delta y_{0}=0.05, \quad \text { and } \quad p_{0}=1500 \text {. }
$$

With these values, $t_{0}=(0.05)^{2}=2.5 \times 10^{-3}$, while $T_{c l}^{(p o)}(\min )=(0.58,1.00) \times 10^{-3}$ and one can see many classical periods for most of the lowest path length classical closed orbits before significant spreading occurs.

In order to probe whether a wave packet has returned to something close to its initial state after some expected classical period, it is standard to examine the autocorrelation function [22], defined, for example, in one-dimension as

$$
A(t) \equiv \int_{-\infty}^{+\infty} \psi^{*}(x ; t) \psi(x ; 0)=\sum_{n=1}^{\infty}\left|a_{n}\right|^{2} e^{i E_{n} t / \hbar}
$$

which also explicitly depends on the energy spectrum. Evaluating $A(t)$ for our twodimensional wave packets, we then plot, in Fig. 8, $|A(t)|$ versus $t / \tau$ (where $\tau \equiv a / v_{0}$ ) for Gaussian wave packets which are initially located at the geometric center of the triangular well $\left(\left(x_{0}, y_{0}\right)=(0, \sqrt{3} a / 3)\right)$, and with the initial momentum in the $\theta$ direction: we then show results for various $\theta$ values in the range $0^{\circ} \leq \theta \leq 30^{\circ}$. (Because of the symmetry of the billiard, such plots will be symmetric about $\theta=30^{\circ}$.)

We expect to see features in $A(t)$ at values of $\theta$ and classical periods $L(p, q) / v_{0}$ given in Table I as we 'scan' up in angle. The stars in Fig. 8 denote the location (in $\left(\theta, T_{c l}^{(p o)}\right)$ parameter space) where we would expect classical particles to return to their starting point in phase space and we do indeed see well-localized structures in $A(t)$ near each expected location. (The dotted curves which connect the 'stars' representing the exact closed orbits are simply there to guide the eye.) We see this same pattern if we initially localize the Gaussian wave packet to places in the well other than at the geometrical center, corresponding to the infinite sets of non-isolated classical orbits which all have the same path length.

One exception to this occurs for parameters near the initial location $\left(x_{0}, y_{0}\right)=(0, \sqrt{3} a / 4)$ and with $\theta=0$ and the results for $A(t)$ for such this case are shown (on the bottom line) 
as the dotted peaks. They coincide with the standard case, but also exhibit structure at classical path lengths given by odd multiples of $3 a / 2$ and are due to the special isolated $(\bar{i}, \bar{j})=(2.0)^{\prime}$ path shown in Fig. $4(\mathrm{c})$.

Such wave packet solutions can also be constructed in the 'half-well' case, using the ap-

propriately normalized versions of the $\psi_{(m, n)}^{(-)}(x, y)$. As mentioned above, an initial Gaussian wave packet will be expandable (to exponential accuracy) in this way, provided it is contained within an appropriate 'fiducial' area of the triangle, well away from any of the boundaries. We note that because such a wave packet would also be expandable in the original 'full-well' (with coefficients given by Eqn. (54)), the expansion coefficients in the 'half-well', $\tilde{a}_{(m, n)}^{(-)}$, will necessarily satisfy the relation

$$
\tilde{a}_{(m, n)}^{(-)}=\sqrt{2} a_{(m, n)}^{(-)}
$$

Since the $\tilde{a}_{(m, n)}^{(-)}$must saturate the complete probability for the 'half-well' version of the wave packet, we must then have

$$
1=\sum_{m>2 n}^{\infty} \sum_{n=1}^{\infty}\left|\tilde{a}_{(m, n)}^{(-)}\right|^{2}=2 \sum_{m>2 n}^{\infty} \sum_{n=1}^{\infty}\left|a_{(m, n)}^{(-)}\right|^{2}
$$

and the odd expansion coefficients (for such wave packets which are well inside one half of the original 'full well') give exactly half of the total probability. For wave packets in the 'half-well', the only new closed orbit path length feature, beyond those seen in Fig. 8, arises from wave packets which are constructed to mimic the special isolated $(1,1)^{\prime}$ orbit in Fig. 6(c).

\section{Long-term revival behavior}

The long-term behavior of wave packets can include the possibility of revivals, wherein initially localized states, after undergoing a number of semi-classical periods eventually spread out during a so-called collapsed phase, only to reform later into something very much like the initial state. The time scales governing these revivals, in a two-quantum number system are given by [12], [13]

$$
T_{\text {rev }}^{(m)}=\frac{2 \pi \hbar}{\left(\partial^{2} E / \partial m^{2}\right) / 2} \quad, \quad T_{r e v}^{(n)}=\frac{2 \pi \hbar}{\left(\partial^{2} E / \partial n^{2}\right) / 2} \quad, \quad T_{r e v}^{(m, n)}=\frac{2 \pi \hbar}{\left(\partial^{2} E / \partial m \partial n\right)} .
$$


For the equilateral triangle under consideration here, we have the simple result

$$
T_{\text {rev }}^{(m)}=T_{r e v}^{(n)}=T_{r e v}^{(m, n)} \equiv T_{r e v}=\frac{9 \mu a^{2}}{4 \hbar \pi}
$$

and exact revivals (just as for the 2D square well) are present in this system, with a single revival time guaranteed for any and all possible initial wave packets.

To visualize this phenomenon, we again make use of Gaussian wave packets, with the same parameters used above, but with vanishing momentum $\left(p_{0}=0\right)$ where there would be no obvious classical periodicity. We locate several such wave packets at initial positions (keeping away from the edges) along the $y$-axis, as shown in Fig. 9, and plot the autocorrelation function over a time equal to one revival time, $T_{r e v}$. In each case we do find an exact revival at the expected time, but we also notice special situations with exact revivals at shorter times, due to additional symmetries of the eigenfunctions, which result in the vanishing of certain expansion coefficients. For example, for $\left(x_{0}, y_{0}\right)=(0, \sqrt{3} a / 3)$ (geometric center of the well), one finds revivals at multiples of $T_{\text {rev }} / 9$ since only expansion coefficients for which $(m+n)$ is a multiple of 3 are non-vanishing, due to the nodal pattern of the eigenstates superimposed on the circular symmetry of the $\left(p_{0 x}, p_{0 y}\right)=(0,0)$ Gaussian wavefunction; in a similar fashion, if $\left(x_{0}, y_{0}\right)=(0, \sqrt{3} / 4)$ (initial position half way down the bisector), one finds exact revivals at multiples of $T_{r e v} / 4$. Even at these special points, if the momentum is made non-vanishing, only the true revivals at $T_{\text {rev }}$ remain intact, since the purely radially symmetric nature of the wavefunctions is lost. (Similar cases such as these are also seen in the $1 \mathrm{D}$ and 2D square well [14] for zero-momentum wave packets when obvious symmetries dictate that certain subsets of expansion coefficients vanish.)

Finally, since the 'half-well' version of the triangular well also has integrally related energies, exact revivals are also present in the $30^{\circ}-60^{\circ}-90^{\circ}$ triangle, but without the special cases mentioned above present.

\section{CONCLUDING REMARKS}

We have used the triangular billiard, with its simple energy spectrum, as a model system to probe periodic orbit theory and wave packet revivals. Similarly to the cases of the square $(N=4$ polygon) and circular $(N \rightarrow \infty$ polygon $)$ billiards, we find that for the equilateral triangular $(N=3)$ infinite well the periodic orbit theory analysis agrees well 
with expectations, including special isolated orbits for various 'folded' versions; for the case here, we find a special isolated orbit in the 'full-well' version as well. The short-term, semi-classical propagation of quantum wave packets shows evidence, in the autocorrelation function, of the same closed orbits, including the isolated ones.

The equilateral triangle billiard also exhibits exact wave packet revivals (due to the integral nature of the energy eigenvalues), just like the 2D square billiard, as does the 'halfwell' version, for all wave packets.

The case considered here might also be used as a basis for a more systematic examination of the quantum energy spectrum of the $N=6$ polygon, namely the hexagonal billiard. The solutions here will form a subset of the allowed spectrum of the $N=6$ case and an examination of both the periodic orbits and wave packet revivals in such a system will require the complete enumeration of the energy eigenvalues, either in closed form, or perhaps using various approximation methods 23] : having a subset of energy eigenvalues and wavefunctions for which the results are known exactly would, of course, provide a very useful 'benchmark' for any numerical calculation. That case would also be another interesting example of a $\pi / N$ billiard 24]. Such a result would be useful in probing the pattern of wave packet revivals as one goes from the $N=3,4$ cases (where exact revivals are present) to the $N \rightarrow \infty$ (circular) case, where only approximate revivals exist.

\section{Acknowledgments}

The work of R. W. R. was supported, in part, by the National Science Foundation under Grant DUE-9950702. The work of M. A. D. was supported, in part, by the Commonwealth College of the Pennsylvania State University under a Research Development Grant (RDG).

\section{APPENDIX A}

In Sec. II(c) we exhibited several simple transformations of the $(m, n)$ quantum numbers of the equilateral triangular well which, when applied to allowed $(m, n)$ pairs, returned quantum numbers and corresponding energy eigenvalues appropriate to higher energy states which, in turn, could be easily visualized in terms of simple 'foldings' of the half-well. For example, the maps $\left(m^{\prime}, n^{\prime}\right)=(f m, f n)$ gave energies which are $f^{2}$ times larger, correspond- 
ing to $f^{2}$ smaller 'copies' of the initial $(m, n)$ state inside the full triangular well. The less obvious mapping given by $\left(m^{\prime}, n^{\prime}\right)=(2 m-n, m-2 n)$ gave energies which were three times larger, corresponding to obvious 'foldings' in thirds, as evident from the resulting wave function plots, as in Fig. 2.

If we define a dimensionless function, $\epsilon(m, n)$, via

$$
E(m, n) \equiv E_{0} \epsilon(m, n)=E_{0}\left(m^{2}-m n+n^{2}\right)
$$

where $E_{0} \equiv\left(\hbar^{2} / 2 \mu a^{2}\right)(4 \pi / 3)^{2}$, we can see that

$$
\epsilon(f m, f n)=f^{2} \epsilon(m, n) \quad \text { and } \quad \epsilon(2 m-n, m-2 n)=3 \epsilon(m, n) .
$$

This second mapping is a special case of a general set of such mappings which return subsets of the allowed energy eigenvalue space. If we pick any pair of integers, $(p, q)$, of the same form $(p>2 q)$ as the allowed quantum numbers themselves, and define the transformation

$$
\left(m^{\prime}, n^{\prime}\right)=T_{(p, q)}[m, n] \equiv(p m-q n,(p-q) m-p n)
$$

one can easily confirm that

$$
\epsilon\left(m^{\prime}, n^{\prime}\right)=\left[p^{2}-p q+q^{2}\right]\left(m^{2}-m n+n^{2}\right)=\epsilon(p, q) \epsilon(m, n)
$$

and we find all such energies in the spectrum of the equilateral triangle. Two consecutive applications of the same transformation then give

$$
\left(m^{\prime \prime}, n^{\prime \prime}\right)=T_{(p, q)}\left[T_{(p, q)}[m, n]\right]=\left(\left(p^{2}-p q+q^{2}\right) m,\left(p^{2}-p q+q^{2}\right) n\right)
$$

so that

$$
\epsilon\left(m^{\prime \prime}, n^{\prime \prime}\right)=[\epsilon(p, q)]^{2} \epsilon(m, n)
$$

One can similarly show that two successive transformations with different labels, in either order, give rise to equivalent sets of quantum numbers in the sense that the corresponding energies are identical, namely if

$$
\left(m^{\prime \prime}, n^{\prime \prime}\right)=T_{(p, q)}\left[T_{(r, s)}[m, n]\right] \quad \text { or } \quad\left(m^{\prime \prime}, n^{\prime \prime}\right)=T_{(r, s)}\left[T_{(p, q)}[m, n]\right]
$$

both give

$$
\epsilon\left(m^{\prime \prime}, n^{\prime \prime}\right)=[\epsilon(p, q) \epsilon(r, s)] \epsilon(m, n)
$$


which also appear in the original energy spectrum. We note that in order to see that the $\left(m^{\prime \prime}, n^{\prime \prime}\right)$ given in the two orderings are, in fact, identical, one sometimes must use the $(m, n) \leftrightarrow(m, m-n)$ equivalence in Eqn. (17). These transformations do not seem to have the same obvious geometrical significance as the $T_{(2,1)}(m, n)$ three way 'folding' or the $\left(m^{\prime}, n^{\prime}\right)=(f m, f n)$ 'copying'. 


\section{Tables}

\begin{tabular}{||c|r|l||c|r|l||}
\hline$(\bar{i}, \bar{j})$ & $\theta(\mathrm{deg})$ & path lengths $(L / a<20)$ & $(\bar{i}, \bar{j})$ & $\theta(\mathrm{deg})$ & path lengths $(L / a<20)$ \\
\hline$(2,0)$ & 0.0 & $3.00,6.00,9.00,12.00,15.00,18.00$ & $(11,5)$ & 14.7 & 17.06 \\
\hline$(13,1)$ & 2.5 & 19.52 & $(4,2)$ & 16.1 & $6.24,12.49,18.73$ \\
\hline$(11,1)$ & 3.0 & 16.52 & $(9,5)$ & 17.8 & 14.18 \\
\hline$(9,1)$ & 3.7 & 13.53 & $(5,3)$ & 19.1 & $7.94,15.87$ \\
\hline$(7,1)$ & 4.7 & 10.53 & $(11,7)$ & 20.2 & 17.58 \\
\hline$(12,2)$ & 5.5 & 18.08 & $(6,4)$ & 21.1 & $9.64,19.28$ \\
\hline$(5,1)$ & 6.6 & $7.55,15.10$ & $(7,5)$ & 22.4 & 11.36 \\
\hline$(13,3)$ & 7.6 & 19.67 & $(8,6)$ & 23.4 & 13.08 \\
\hline$(8,2)$ & 8.2 & 12.12 & $(9,7)$ & 24.2 & 14.80 \\
\hline$(11,3)$ & 8.9 & 16.70 & $(10,8)$ & 24.8 & 16.5 \\
\hline$(3,1)$ & 10.9 & $4.58,9.16,13.75,18.33$ & $(11,9)$ & 25.3 & 18.24 \\
\hline$(13,5)$ & 12.5 & 19.97 & $(12,10)$ & 25.7 & 19.97 \\
\hline$(10,4)$ & 13.0 & 15.39 & $(1,1)$ & 30.0 & $1.73,3.46,5.20,6.93,8.66,10.40$, \\
\hline$(7,3)$ & 14.0 & 10.82 & & & $12.12,13.86,15.59,17.32,19.05$ \\
\hline
\end{tabular}

Table I. Path lengths (from Eqn. (321)) and initial angles (from Eqn. (33)) for closed orbits (and recurrences) with $L / a<20$. 


\section{Figure Captions}

Fig. 1. Geometry of the equilateral triangle billiard (shown in bold) with vertices at $(0,0)$, $(+a / 2, \sqrt{3} a / 2)$, and $(-a / 2, \sqrt{3} a / 2)$.

Fig. 2. Nodal patterns for the lowest-lying energy eigenstates for the 'half triangular' well

(using the $\psi_{(m, n)}^{(-)}(x, y)$ in Eqn. (2) $)$ on the top row. (The white (black) areas correspond to positive (negative) values of the wavefunction.) The bottom row corresponds to $\left(m^{\prime}, n^{\prime}\right)=(2 m, 2 n)$ states which are a factor of 4 larger in energy, while the middle row shows those related via $\left(m^{\prime}, n^{\prime}\right)=(2 m-n, m-2 n)$ which are a factor of 3 higher in energy, illustrating various 'foldings' of the half-well.

Fig. 3. Geometrical visualization leading to the construction of the path lengths corresponding to various closed orbits in the triangular billiard.

Fig. 4. Closed orbits corresponding to several $(\bar{i}, \bar{j})=(1,1)$ cases (bottom row) with path lengths given by multiples of $\sqrt{3} a$ and for $(\bar{i}, \bar{j})=(2,0)$ cases (top row) with path lengths given by multiples of $3 a$, except for (c) which is an isolated orbit (described as $(2,0)^{\prime}$ in the text) with path length given by $3 a / 2$ (and multiples thereof.)

Fig. 5. Plot of $\left|\rho_{N}(L)\right|^{2}$ versus $L / a$ for the triangular billiard, evaluated using the 1000 lowestlying energy eigenvalues. All of the expected path length features from Eqn. (32) and Table I are indicated by vertical dotted lines. The small bumps indicated by arrows correspond to the isolated $(2,0)^{\prime}$ orbits in Fig. 4(c) with path lengths given by multiples of $3 a / 2$.

Fig. 6. Closed orbits in the 'half-triangle' well for the $(\bar{i}, \bar{j})=(1,1)$ case. For (a) and (b), the path lengths are still given by $L(1,1)=\sqrt{3} a$ (and multiples thereof), but the 'folding' induces a single isolated $(1,1)^{\prime}$ orbit with $L=\sqrt{3} a / 2$ as shown in (c).

Fig. 7. Plot of $\left|\rho_{N}(L)\right|^{2}$ versus $L / a$, for the triangular billiard (dashed curve) and the 'halftriangle' billiard (solid curve). Vertical dashed lines indicate the expected path length features in Table I, while the vertical arrows indicate the small new features (at $L / a=$ $\sqrt{3} / 2$ and multiples thereof) present in the 'half-triangle' due to the new, isolated closed orbit in Fig. 6(c). 
Fig. 8. Autocorrelation function, $|A(t)|$, versus $t / \tau$, (where $\tau \equiv a / v_{0}$ ) for Gaussian wave packets with the parameters discussed in Eqn. (59). Wavepackets initially localized at $\left(x_{0}, y_{0}\right)$ corresponding to the geometric center of the triangle are given momenta $\left(p_{0} \cos (\theta), p_{0} \sin (\theta)\right)$ and $|A(t)|$ plots are shown for $\theta$ values in the range $\left(0^{\circ}, 30^{\circ}\right)$. The 'stars' indicate the location of classical closed orbits (corresponding to the values in Table I). The dotted $|A(t)|$ data for the $\theta=0^{\circ}$ case corresponds to the initial wave packet localized at $\left(x_{0}, y_{0}\right)=(0, \sqrt{3} a / 4)$ which corresponds classically to the isolated $(2,0)^{\prime}$ orbit in Fig. 4(c).

Fig. 9. Autocorrelation function, $|A(t)|^{2}$ versus $t$, for zero-momentum Gaussian wave packets initialized at various points along $x=0$ in the triangular well. There are exact revivals for all cases shown (where $\left|A\left(T_{\text {rev }}\right)\right|=1$ ), but also special, shorter-period revivals at multiples of $T_{\text {rev }} / 9$ and $T_{\text {rev }} / 4$ for wave packets initially at positions of special symmetry in the well, namely at the geometric center $(0, \sqrt{3} a / 3)$ (square box) and at $(0, \sqrt{3} a / 4)$ (half way down the bisector.) 
[1] M. Gutzwiller, "Chaos in Classical and Quantum Mechanics", Spring-Verlag, Berlin, 1990.

[2] M. Gutzwiller, "The Interplay Between Classical and Quantum Mechanics", American Association of Physics Teachers, College Park, MD, 2001.

[3] M. Brack, and R. K. Bhaduri, "Semiclassical Physics", Addison-Wesley, Reading, MA, 1997.

[4] G. Alber and P. Zoller, Phys. Rep. 199 (1991), 231.

[5] For Rydberg atoms, Z. D. Gaeta, M. W. Noel, and C. R. Stroud, Jr. Phys. Rev. Lett. 73 (1994), 636; for Stark wave packets, R. Bluhm, V. A. Kosteleckỳ, and B. Tudose, Phys. Rev. A55 (1997), 819; for alkali-metal atoms, R. Bluhm and V. A. Kostelecký, Phys. Rev. A51 (1995), 4767; for the Jaynes-Cummings model, I. Sh. Averbukh, Phys. Rev. A46 (1992), R2205; for wave packets in a Morse-type potential well, S. I. Vetchinskin and V. V. Eryomin, Chem. Phys. Lett. 222 (1994), 394; for an atom in a gravity well; W. -Y. Chen and G. J. Milburn, Phys. Rev. A51 (1995), 2328; J. Gea-Banacloche, Am. J. Phys. 67 (1999), 776.

[6] M. C. Gutzwiller, J. Math. Phys. 11 (1970), 1791.

[7] R. Balian and C. Bloch, Ann. Phys. 69 76, (1972).

[8] H.-J. Stöckmann and J. Stein, Phys. Rev. Lett. 64 (1990), 2215; E. Doron, U. Smilansky, and A. Frenkel, Phys. Rev. Lett. 65 (1990), 3072; S. Sridhar and E. J. Heller, Phys. Rev. A46 (1992), R1728.

[9] C. M. Marcus, A. J. Rimberg, R. M. Westervelt, P. F. Hopkins, and A. C. Gossard, Phys. Rev. Lett. 69 (1992), 506.

[10] N. Friedman, A. Kaplan, D. Carasso, and N. Davidson, Phys. Rev. Lett. 86 (2001), 1518.

[11] D. L. Aronstein and C. R. Stroud Jr, Phys. Rev. A55 (1997), 4526; P. Stifter, W. E. Lamb Jr, and W. P. Schleich, in "Frontiers of Quantum Optics and Laser Physics", Proceedings of the International Conference on Quantum Optics and Laser Physics, (S. Y. Zhu, M. S. Zubairy, and M. O. Scully, Eds.), pp. 236-246, Springer-Verlag, Singapore, 1997.

R. Bluhm, V. A. Kosteleckÿ, and J. Porter, Am. J. Phys. 64 (1996), 944; F. Großmann, J. -M. Rost, and W. P. Schleich, J. Phys. A30 (1997), L277; R. W. Robinett, Am. J. Phys, 68 (2000), 410; D. F. Styer, Am. J. Phys. 69 (2001), 56.

[12] R. Bluhm, V. Alan Kostelecký, and B. Tudose, Phys. Lett. A222 (1996), 220.

[13] G. S. Agarwal and J. Banerji, Phys. Rev. A57 (1998), 3880. 


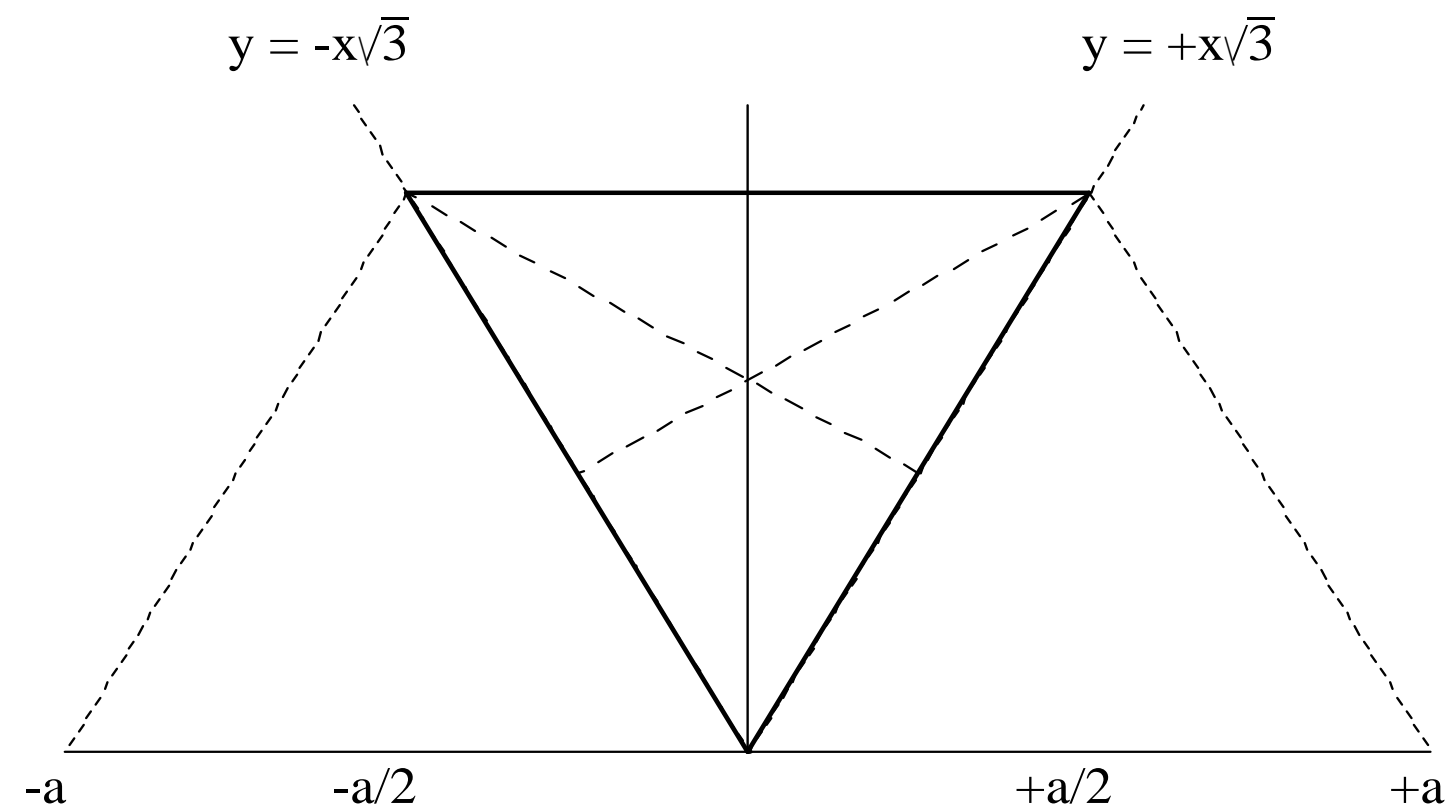

FIG. 1:

[14] R. W. Robinett and S. Heppelmann, "Quantum wave packet revivals in circular billiards", PSU/TH/250 (to appear in Phys. Rev. A.)

[15] C. Jung, Canadian Journal of Physics, 58 (1980), 719. This paper discusses the group theoretical properties of the solutions of the triangular well in some detail.

[16] P. J. Richens and M. V. Berry, Physica 2D (1981), 495.

[17] A version of this problem is also discussed in J. Mathews and R. L. Walker, "Mathematical Methods of Physics", 2nd Edition, pp. 237-239, W. A. Benjamin, Menlo Park, 1970.

[18] R. W. Robinett, J. Math. Phys. 40 (1999), 101.

[19] M. V. Berry and M. Wilkinson, Proc. Roy. Soc. London, Ser. A 392 (1984), 15.

[20] R. W. Robinett, Am. J. Phys. 65 (1997), 1167.

[21] K. Richter, D. Ullmo, and R. A. Jalabert, Phys Rep. 276 (1996), 1.

[22] M. Nauenberg, J. Phys. B. At. Mol. Opt. Phys. 23 (1990), L385.

[23] The equilateral triangle billiard has also been used as a 'testbed' for approximation methods for the calculation of eigenstates of quantum billiards as in D. L. Kauffman, I. Kosztin, and K. Schulten, Am. J. Phys. 67 (1999), 133.

[24] A. Hobson, J. Math. Phys. 16 (1975), 2210. 

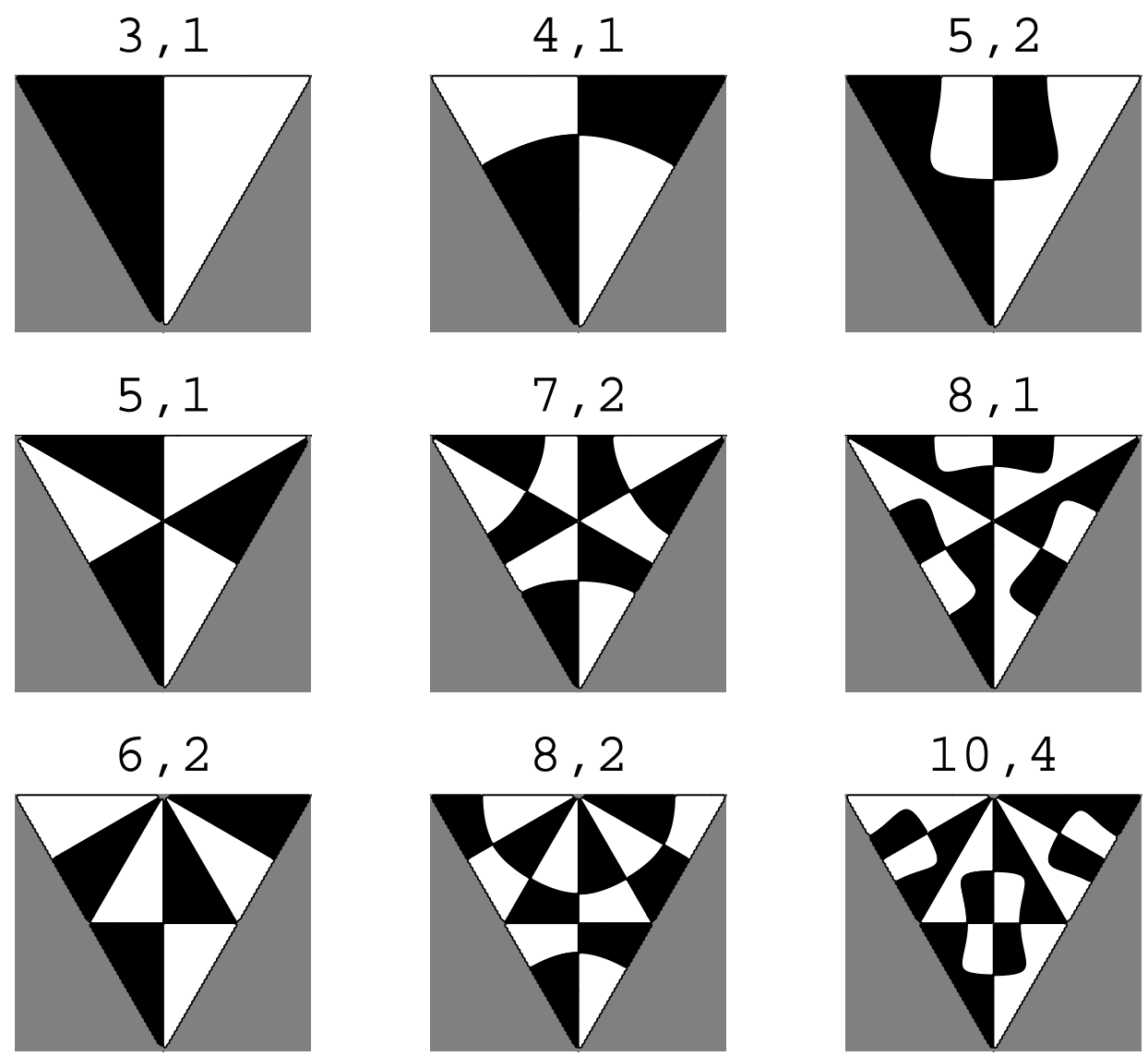

FIG. 2: 


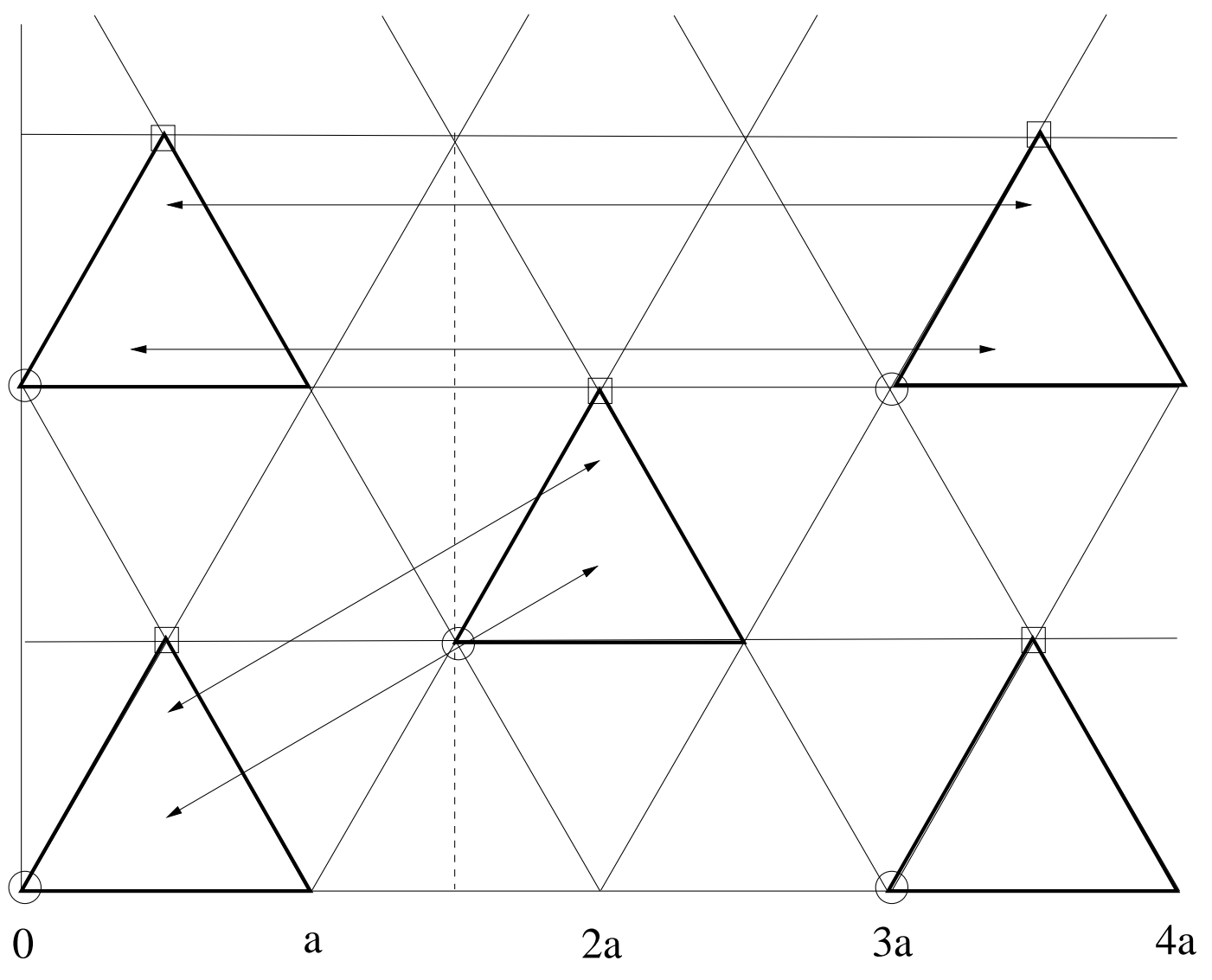

FIG. 3: 


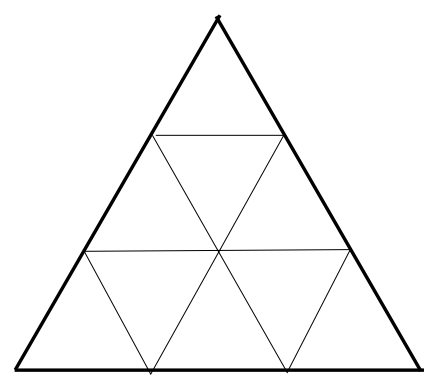

(a)

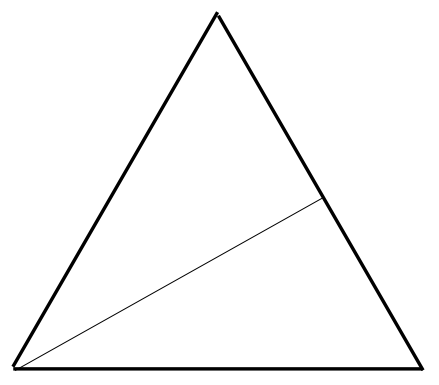

(d)

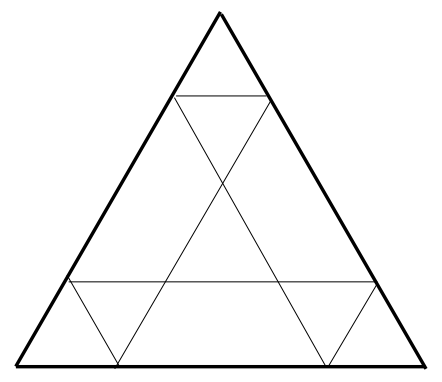

(b)

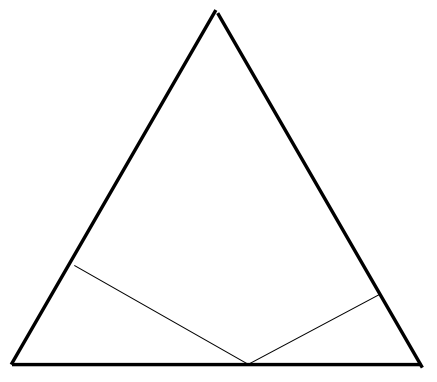

(e)

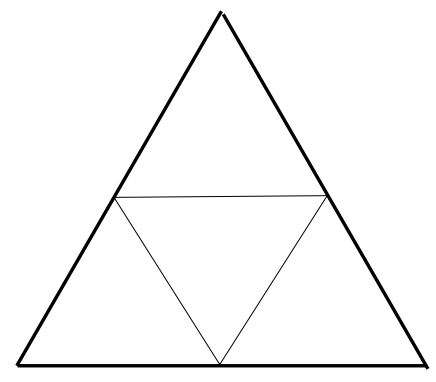

(c)

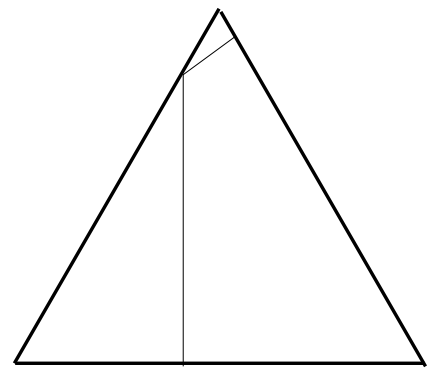

(f)

FIG. 4: 


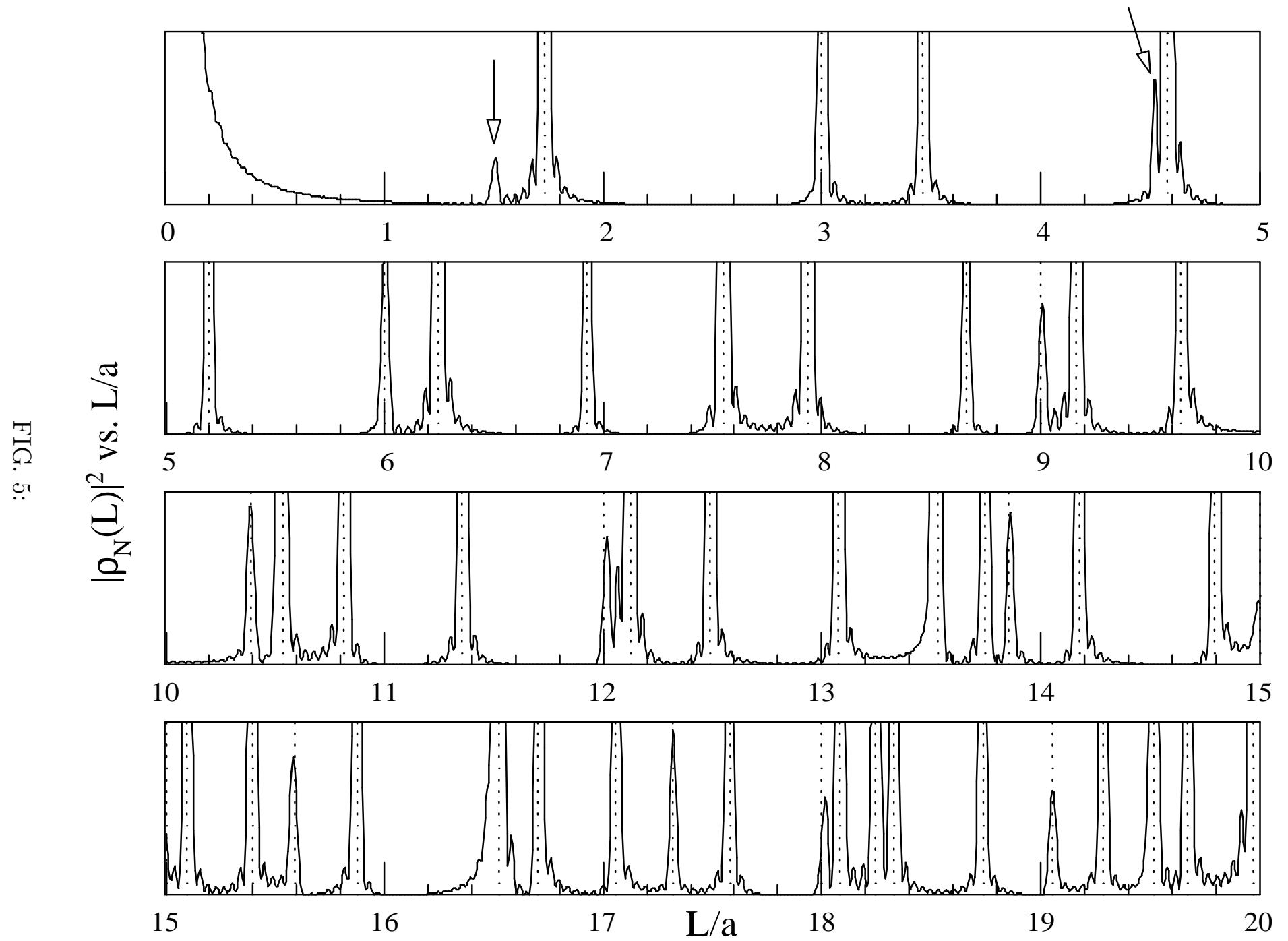




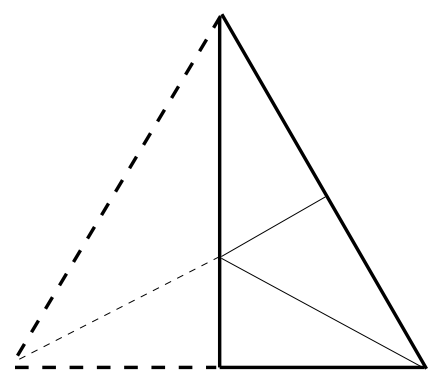

(a)

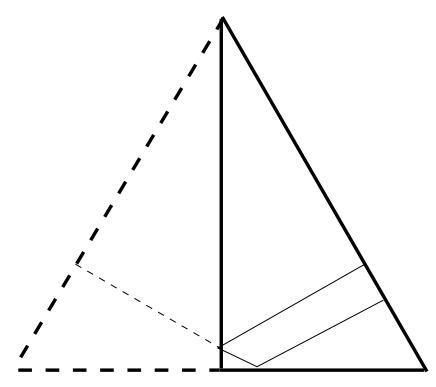

(b)

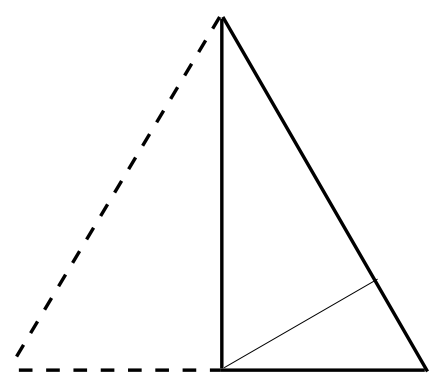

(c)

FIG. 6: 


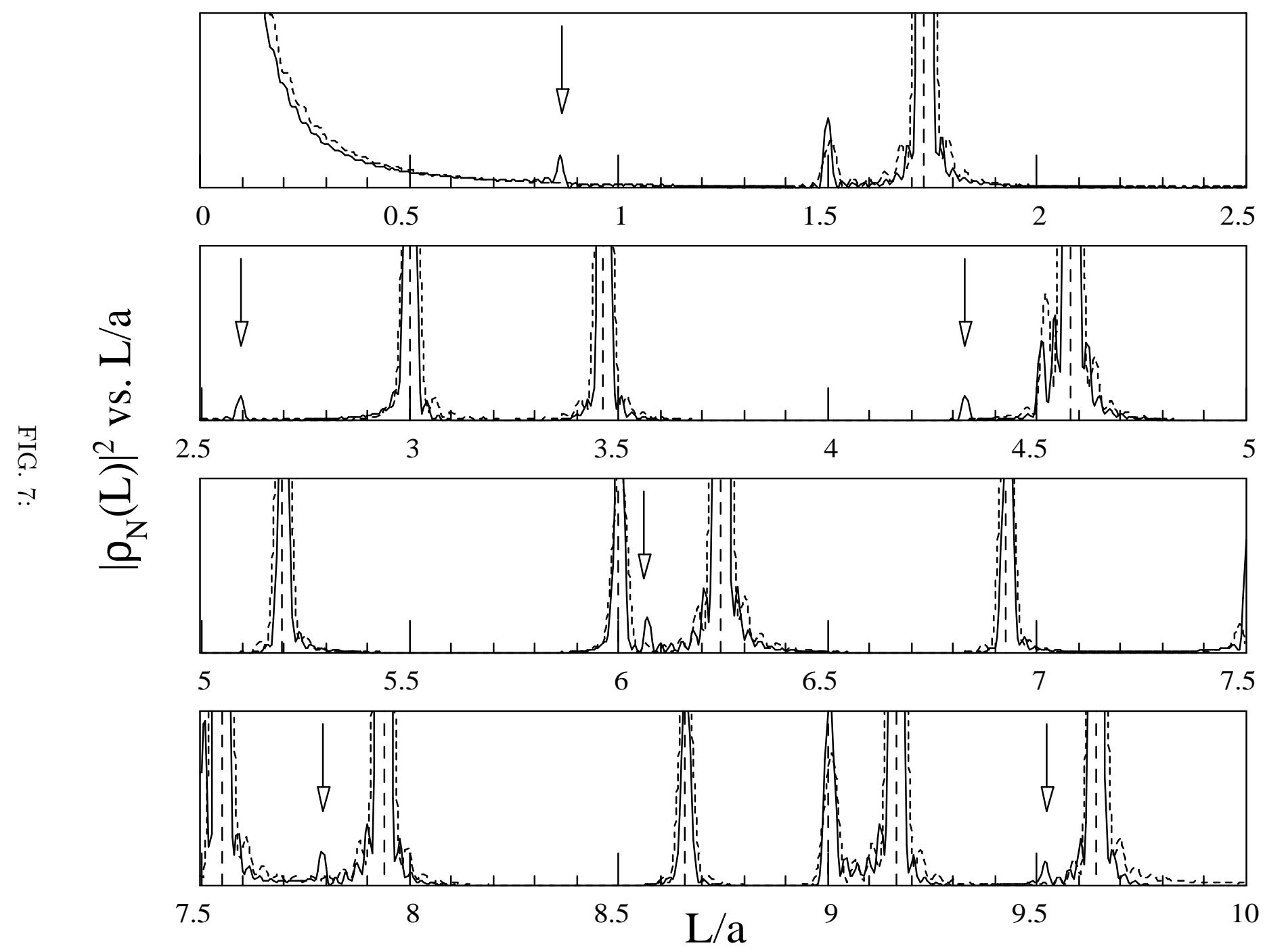




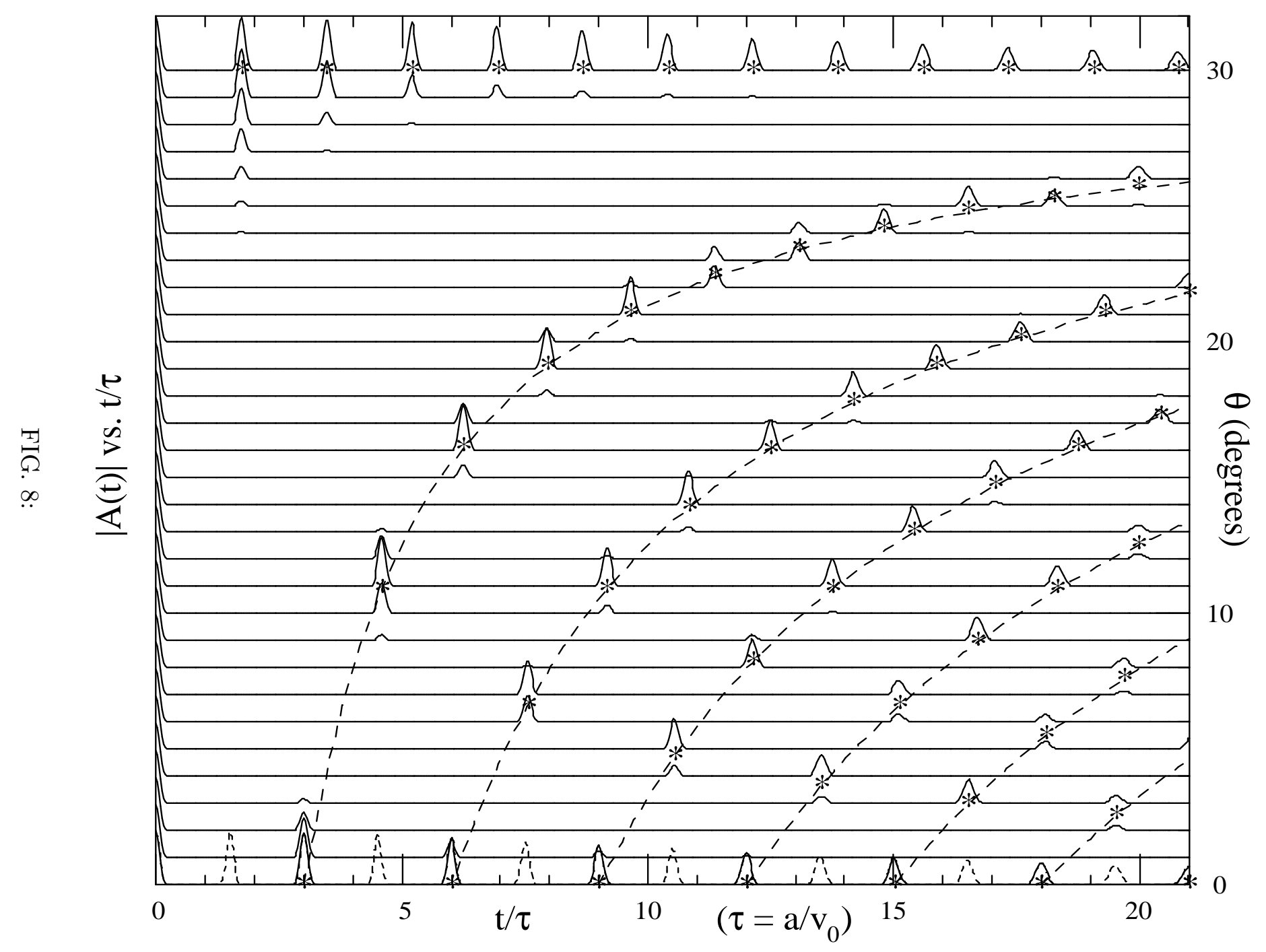




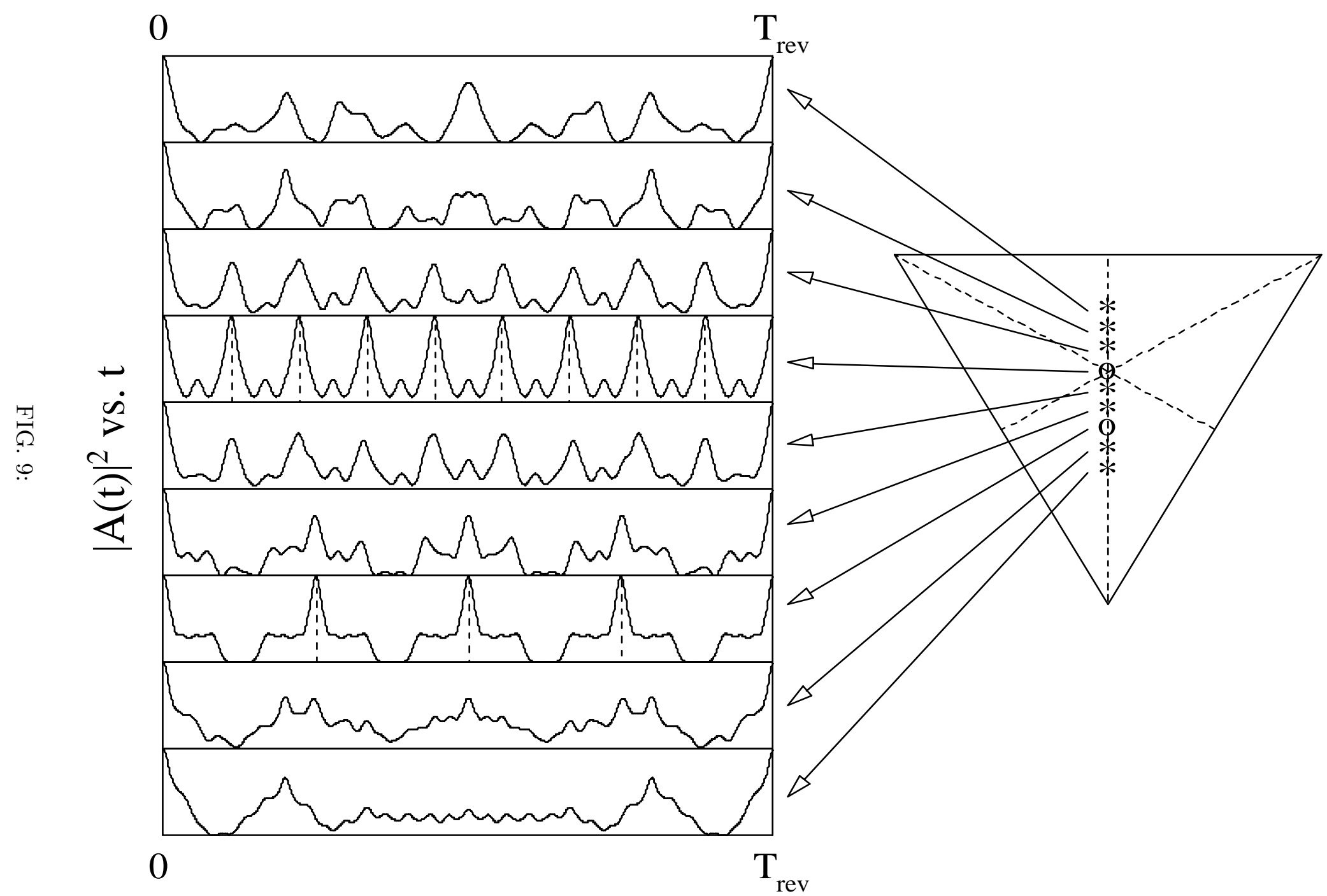

